Supporting Information

\title{
Inhibition of the Proteolytic Activity of Anthrax Lethal Factor by Aminoglycosides
}

Lac V. Lee, Kristen E. Bower, Fu-Sen Liang, Jin Shi, Douglass Wu, Steven J. Sucheck, Peter K. Vogt,* and Chi-Huey Wong*

Department of Chemistry and the Skaggs Institute for Chemical Biology; Department of Molecular and Experimental Medicine, Division of Oncology; The Scripps Research Instiute, 10550 Torrey Pines Road, La Jolla, California 92037. Optimer Pharmaceuticals, Inc., 10110 Sorrento Valley Rd, Suite C, San Diego, CA 92121.

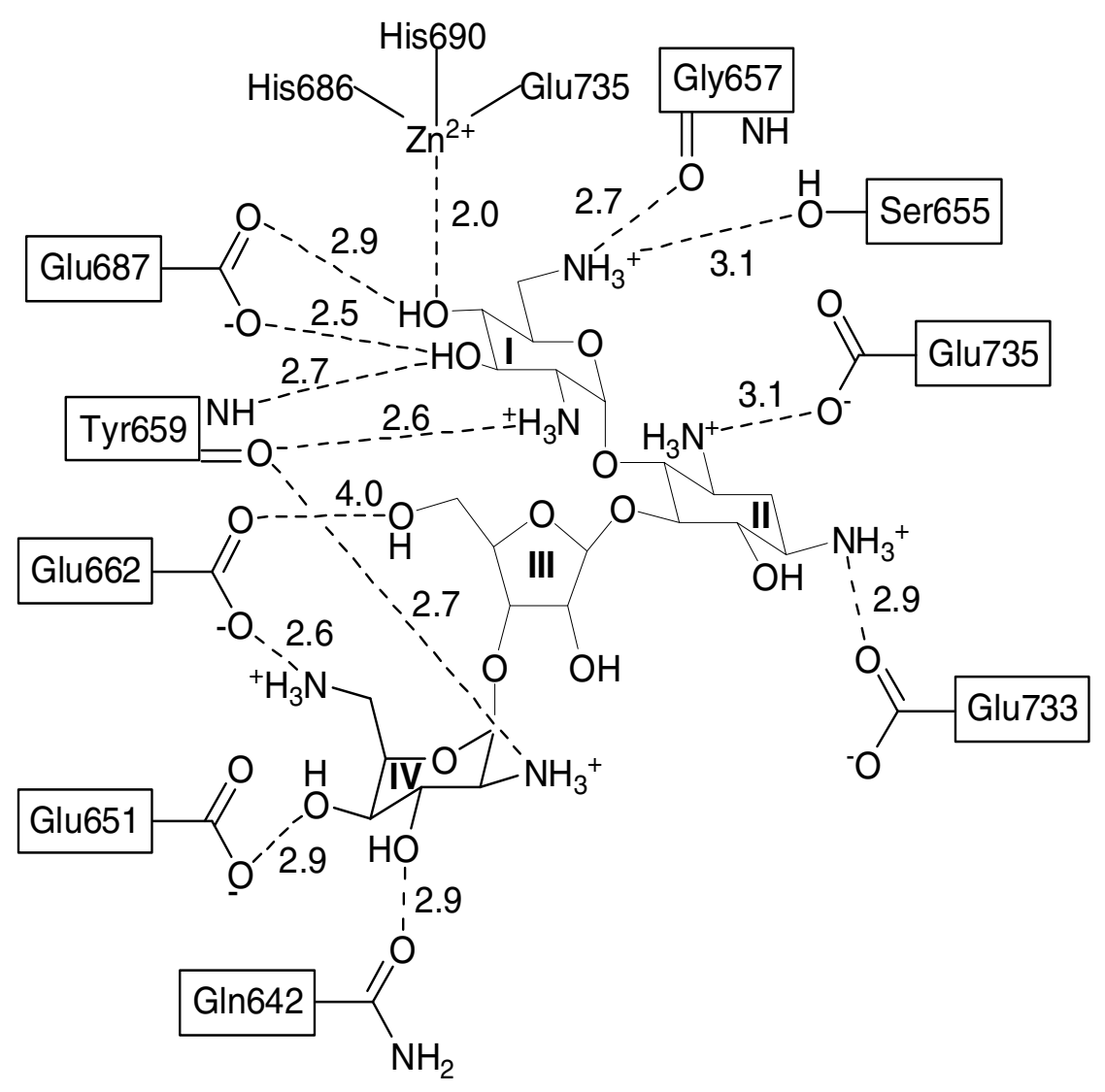

NeomycinB/LF complex. Distances are measured between heteroatoms in angstrom $(\AA)$. 


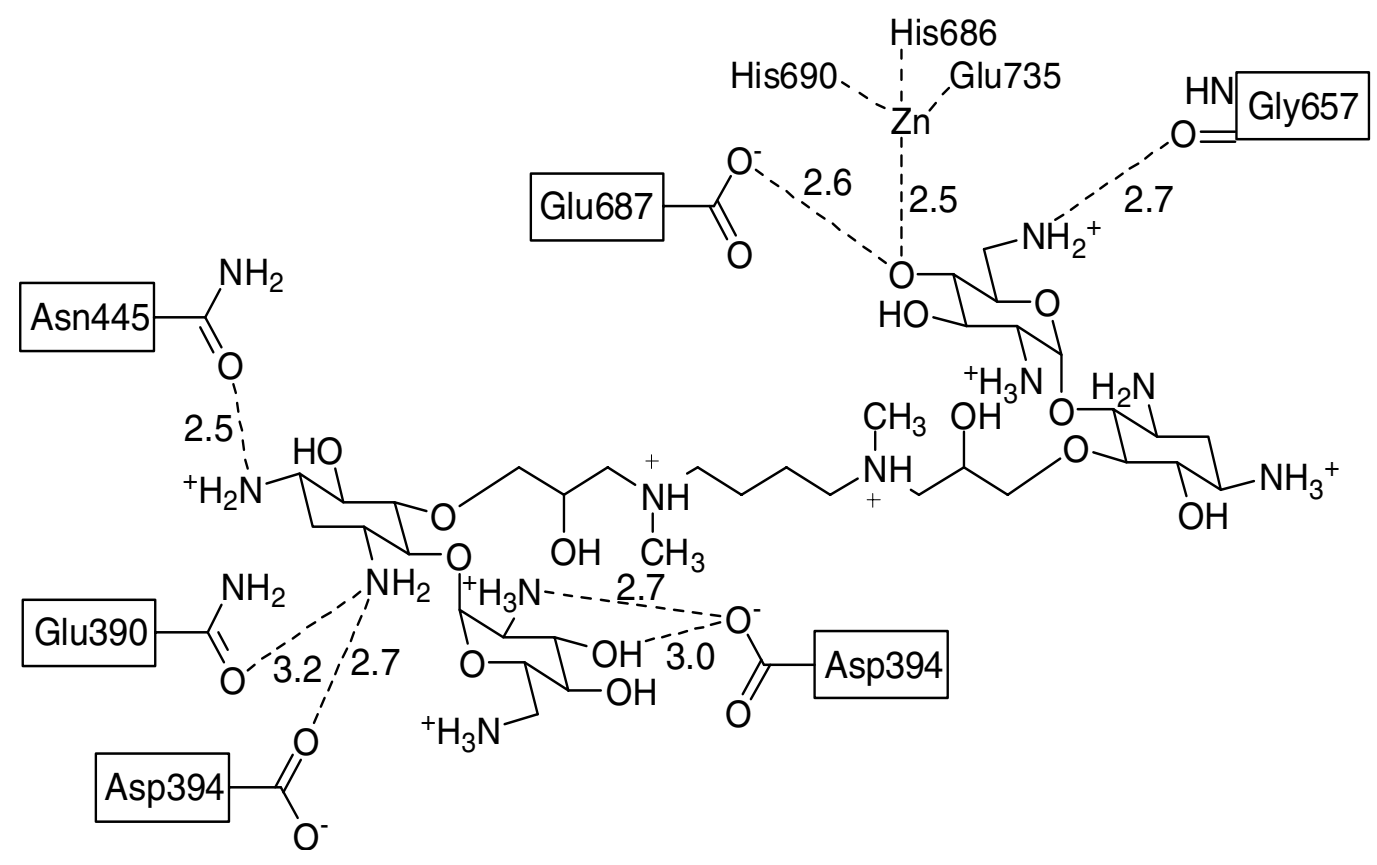

OP71/LF Complex. Distances are measured between heteroatoms in angstrom $(\AA)$. 